\title{
COMMON SPECTRAL FEATURES IN THE 5500-YEAR RECORD OF TOTAL CARBONATE IN SEA SEDIMENTS AND RADIOCARBON IN TREE RINGS
}

\author{
GIULIANA CINI CASTAGNOLI, GIUSEPPE BONINO, MARINA SERIO
}

Istituto di Fisica Generale Università, Istituto di Cosmo-Geofisica, CNR, Corso Fiume 4 10133 Torino, Italy

and

\section{CHARLES P. SONETT}

Department of Planetary Sciences, Lunar and Planetary Laboratory, The University of Arizona Tucson, Arizona 85721 USA

\begin{abstract}
We analyze here the time record of total carbonate carried as $\mathrm{CaCO}_{3}$ in a sea-bottom core from the Ionian Sea. Several major periods appear, most strongly at 1100, 690, 500, 340, 250 and $90 \mathrm{yr}$, confirmed both in the periodogram and maximum entropy estimates. The Gleissberg period appears in common with the ${ }^{14} \mathrm{C}$ and other records, such as the sunspot index and aurorae. The manifestation of this period in what we surmise to be a climatic record is further evidence that the Gleissberg period has correlated bolometric and electrodynamic aspects.
\end{abstract}

\section{INTRODUCTION}

We show here that the time series of total carbonate $\left(\mathrm{as}_{\mathrm{CaCO}}\right)$ deposited in the past $5.5 \mathrm{ka}$ on the continental shelf of the Ionian Sea reveals several quasiperiodic features. One of these is identified with the Gleissberg period normally seen in solar terrestrial electrodynamic parameters, such as the sunspot index, aurorae and the geomagnetic field (Gleissberg 1944, 1966; Feynman \& Fougere 1984).

The core used for this analysis (GT90/3) was taken in the Gulf of Taranto on the Apulian Continental shelf at $39^{\circ} 45^{\prime} 53^{\prime \prime} \mathrm{N}, 17^{\circ} 53^{\prime} 33^{\prime \prime} \mathrm{E}$, water depth, $174 \mathrm{~m}$. Previously, we took Cores GT14 and GT89/3 from this region. The experimental data of carbonate concentration as a function of the time and the analysis of the periodic features found in these series have been reported in previous papers (Cini Castagnoli et al. 1990a, 1990b; Cini Castagnoli, Bonino \& Provenzale 1991). The length of Core GT90/3 is $3.57 \mathrm{~m}$, the longest that we have taken in this region. The sedimentation shows no obvious laminae; dating is based upon evaluation of ${ }^{210} \mathrm{~Pb}\left(\mathrm{~T}_{1 / 2}=22.3 \mathrm{yr}\right)$ "excess" of the isotope with respect to the activity supported in situ by ${ }^{226} \mathrm{Ra}\left(\mathrm{T}_{1 / 2}=1600 \mathrm{yr}\right)$. The "excess" ${ }^{210} \mathrm{~Pb}$ is atmospheric fallout from the decay of ${ }^{222} \mathrm{Rn}\left(\mathrm{T}_{1 / 2}=3.82\right.$ days). Core dating by this method is restricted to ages not greater than $150 \mathrm{yr}$. The correlation coefficient between the profile of "excess" ${ }^{210} \mathrm{~Pb}$ in the sediment and a decreasing exponential provides information on the uniformity of the sedimentation rate over the past two centuries. Checks on the $\mathrm{Pb}$ age profile and its extrapolation to the whole core are obtained from a ${ }^{137} \mathrm{Cs}$ spike at AD $1963-1964$ due to a peak in nuclear weapons testing, on the one hand, and from tephroanalysis, on the other. The latter identifies clinopyroxene sedimentation peaks corresponding to well-known historical volcanic eruptions on Ischia (AD 1301), Pollena (AD 472) and Pompei (AD 79). The Astroni eruption (3600 $\mathrm{BP}$ ) has also been recognized by tephroanalysis. Lastly, comparison of deep-sea Core CT85/5 (not otherwise discussed here) with tephroanalysis of GT90/3 shows a striking correspondence (Cini Castagnoli et al. 1992).

We divided the core into 1427 samples of equal thickness $(\Delta \mathrm{d}=0.25 \mathrm{~cm})$ to determine the total carbonate, represented as $\mathrm{CaCO}_{3}$. Each sample was dried at $80^{\circ} \mathrm{C}$ for two days; the $\mathrm{CaCO}_{3}$ from the powder $(100 \mathrm{mg}$ ) was leached by boiling twice with $20 \mathrm{ml}$ of $2 \%$ acetic acid. We made the 
solution up to $100 \mathrm{ml}$ and after adding a buffer solution, we determined carbonate content by titration with EDTA for each sample, using 3 aliquots of solution. Frequent titration of standard solutions was carried out to insure reproducibility. The triplicate carbonate analyses agreed to within $0.3 \%$.

Figure 1 shows the $\mathrm{CaCO}_{3}$ profiles for Cores GT90/3 and GT14, together with the clinopyroxene volcanic record. The sample interval of $0.25 \mathrm{~cm}$ corresponds to a time interval of $3.87 \mathrm{yr}$. The reference (top) level is AD 1979, and samples are numbered progressively backward in time from this level.

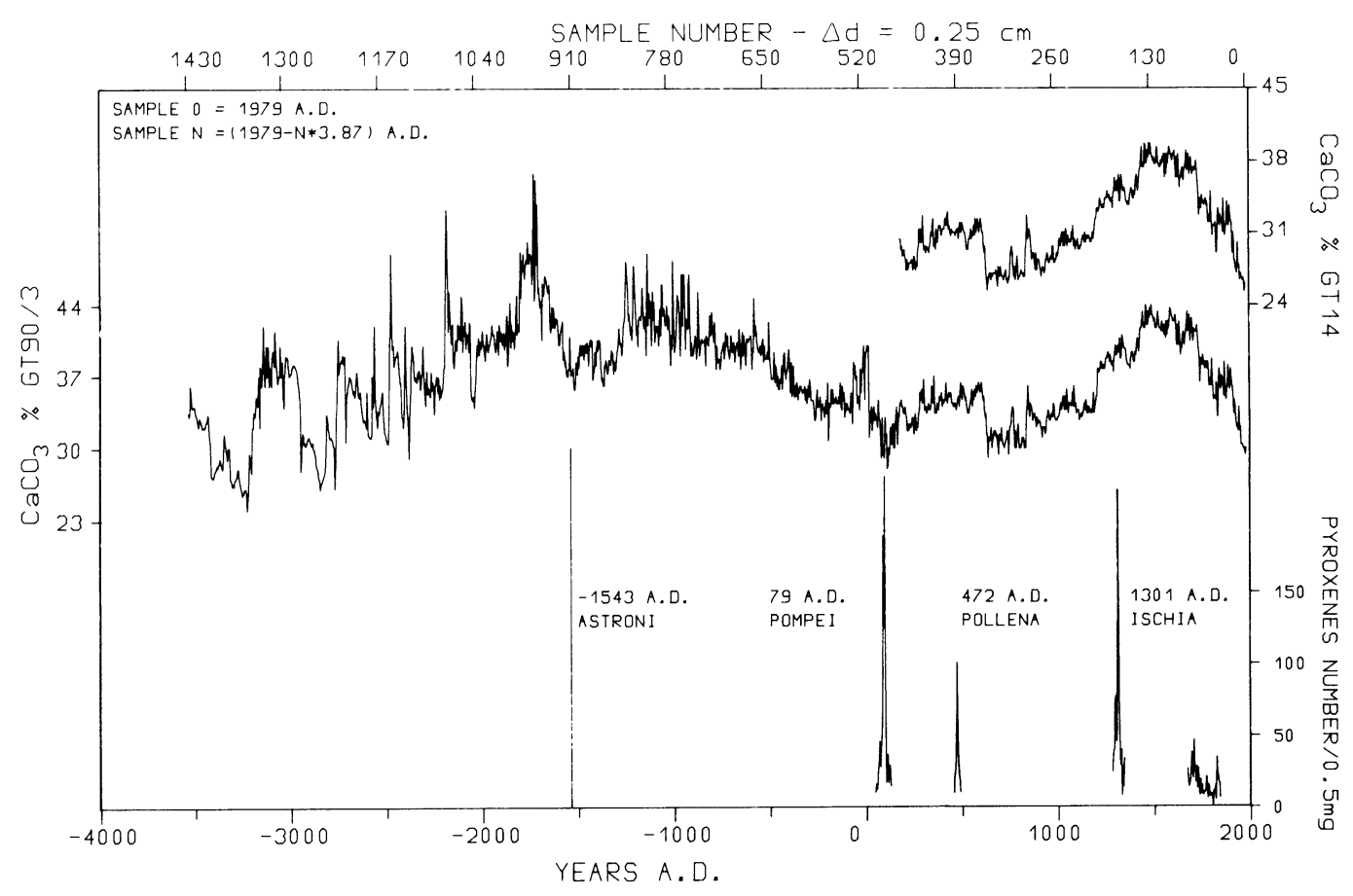

Fig. 1. Carbonate profiles of coastal cores, GT14 and GT90/3, showing the extreme correlation between the carbonate records. Three historic records of pyroxene from volcanic eruptions shown at the base of the figure provide exact time 'benchmarks'.

\section{ANALYSIS OF THE TOTAL CARBONATE HISTORY}

The deposition of $\mathrm{CaCO}_{3}$ depends on its production by organisms and by the meteoric input, plus possible precipitation from $\mathrm{CaCO}_{3}$-saturated sea water and possible dissolution. In turn, all this is dominated by some combination of temperature-dependent solubility, $\mathrm{Ca}^{2+}$, productivity, alkalinity and salinity. These are probably coupled to climate (e.g., Crowley \& North 1991). Relation of the carbonate variability to climatic parameters and ${ }^{14} \mathrm{C}$ rests primarily upon the detection of correlated variations and, specifically, periodicities. We discuss, below, the periodicities recorded in Core GT90/3.

The spatial sample interval, corresponding to a time interval of $3.87 \mathrm{yr}$, is set by laboratory samplesize requirements, which is awkward for use in standard programs and, particularly, for comparison to the ${ }^{14} \mathrm{C}$ record. Thus, the data are interpolated to $1-y r$ intervals, using a cubic spline, and then filtered and decimalized to $10-y r$ intervals on the basis that the Nyquist period is already set at 7.74 


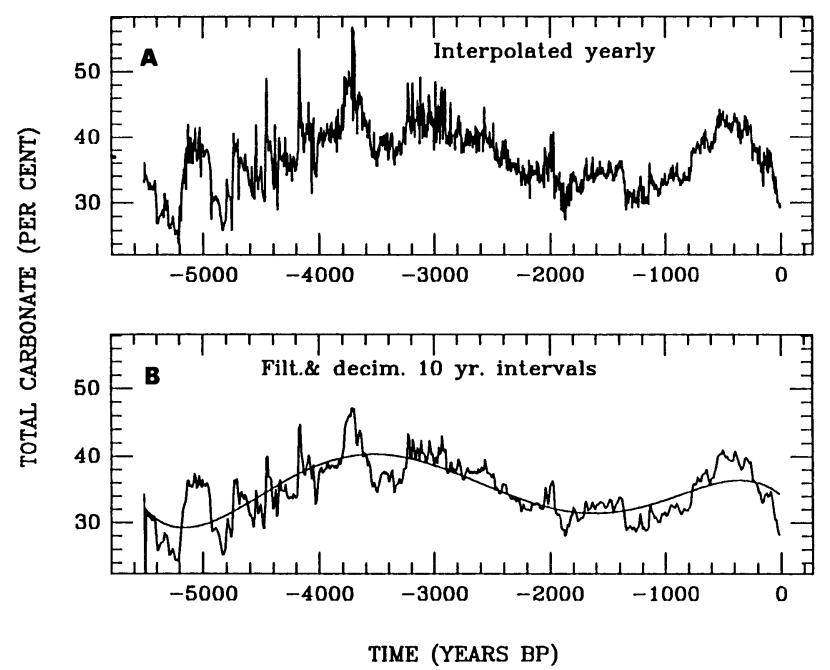

Fig. 2. A. Total carbonate from sea core, GT90/3, interpolated to $1-y r$ intervals. B. Interpolated data filtered to $10-y r$ intervals using a running average. Curve is 5 th-order detrend polynomial.

yr (Figs. 2A, B). For the present, we used simple arithmetic averaging; side-lobe corruption is unimportant for the periods of interest.

\section{Detrending}

The interpolated GT90/3 record disclosed both substantial non-stationarity and long-period variability in the $\mathrm{CaCO}_{3}$ vs. time (Fig. 2A), which, as is well known, must be removed prior to computation of the spectrum. Some or all of the trend might be due to one or more periods lying outside the time span of the data, but these cannot be recovered, except to be treated as a trend. We use a 5th-order polynomial to detrend the record. The alternate sinusoid detrender is probabilistically less likely. Figure $2 \mathrm{~B}$ shows the interpolated data filtered to $10-\mathrm{yr}$ intervals using a running average with the detrend function superimposed. Figure 3 shows the polynomial detrended sequence.

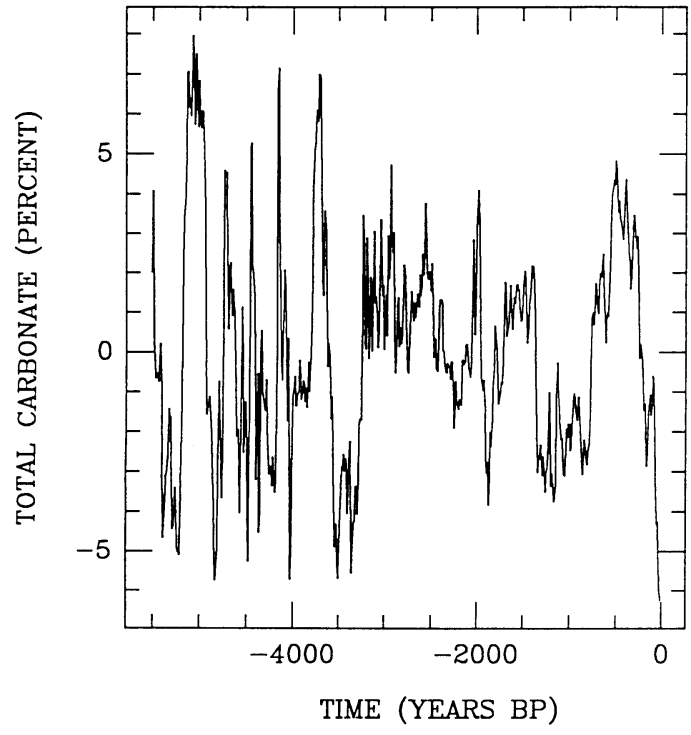

Fig. 3. The total carbonate polynomial detrended and zeromeaned sequence 


\section{The Periodogram and Maximum Entropy (MEM) Spectrum}

Figure 4 shows and Table $1 \mathrm{~A}$ lists the raw unaveraged periodogram from unweighted but detrended and reintervalized carbonate sequence data. The major period is in the neighborhood of $1100 \mathrm{yr}$; multihundred-year lines possibly represent harmonics of the $1100 \mathrm{yr}$, but this is conjectural. That structure is present at higher frequencies is seen from the figure insert showing major features at the Gleissberg period and at about 55-60 yr (not listed in Table 1A), whose source is quite uncertain. It is also noted using MEM (Table 1B). All of these higher frequency lines are very weak compared to the low frequency power, and are detectible only by removing the masking effects of the longer periods.

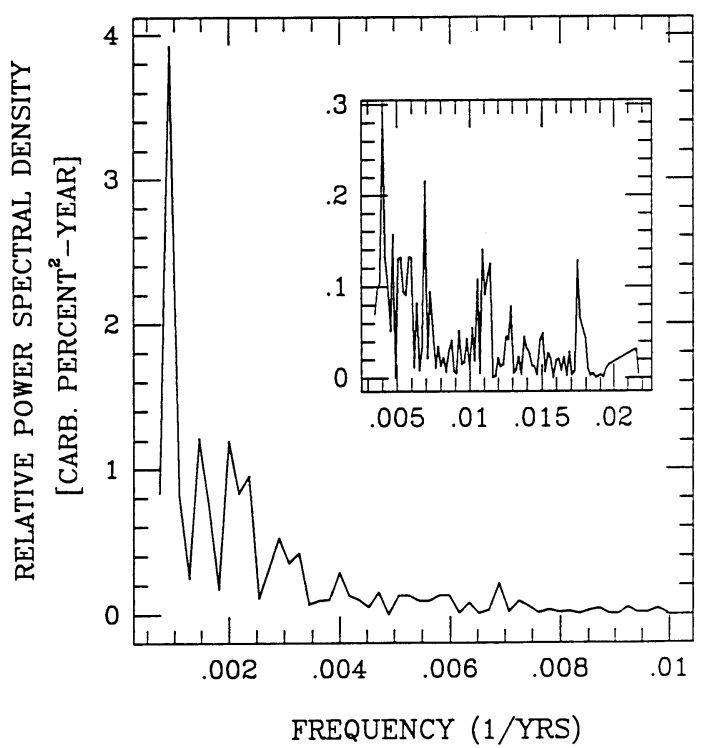

Fig. 4. Unweighted and unfiltered periodogram of GT90/3; insert shows conspicuous minor lines after removal of major features (see also Fig. 5A). Some line-amplitude error is accumulated because spectral features are not computed in an orthogonal space, but this is unimportant for the qualitative analysis done here. The surmise that the multihundred-year cluster of lines is harmonically related to the 1100 -yr feature is strengthened by its approximately equal spacing, emphasized in the inset showing the spectrum less the $1100-\mathrm{yr}$ feature. Moreover, the insert supports the periodogram showing the three low-lying periods present at periods shorter than $100 \mathrm{yr}$, one being identifiable with the Gleissberg line.

Because the periodograms are computed using unweighted data, it is helpful to compare these to the MEM spectrum, which is free of side lobes. Also, MEM is a powerful means of recovering weak low-power spectral features, although spectral shifting and susceptibility to noise are limitations of this technique. Table 1B reports the MEM line listing of the carbonate record. The ${ }^{14} \mathrm{C}$ line listing is given in Table $2 \mathrm{~A}, \mathrm{~B}^{1}$. The carbonate and ${ }^{14} \mathrm{C}$ spectra have some common features, as we discuss now. The frequency resolution is, to some extent, a noise-dependent period. For the periodogram, it is often taken to be $\Delta f=1 / 2 T$, where $T$ is the series length; for MEM the resolution is at least as good.

\section{DISCUSSION}

\section{The Long-Period Regime}

We remark here upon the question of common periodicities in the ${ }^{14} \mathrm{C}$ and total carbonate records. The sea-floor carbonate concentrations result from the $\mathrm{CaCO}_{3}$ deposition processes. A common

\footnotetext{
${ }^{1}$ Some differences between the entries in Table 2A and B and those found earlier, e.g., Sonett and Finney (1990), are attributable to updating of the ${ }^{14} \mathrm{C}$ time sequence. (See Sonett (1992) for the bibliography of the current listing.) The reader should also note that MEM estimates of power are generally of lower accuracy than periodograms.
} 
TABLE 1A. Carbonate Percent Periodogram

\begin{tabular}{ccc}
\hline Period $(\mathrm{yr})^{*}$ & Frequency $\left(\mathrm{yr}^{-1}\right)$ & PDS $\left(\%^{2}-\mathrm{yr}\right)$ \\
\hline $1100 \pm 110$ & 0.0009 & 3.31 \\
$688 \pm 43$ & 0.0015 & 1.10 \\
$500 \pm 23$ & 0.0020 & 1.16 \\
$343 \pm 11$ & 0.0029 & 0.51 \\
$250 \pm 6$ & 0.0040 & 0.24 \\
$90 \pm 1^{* *}$ & 0.0111 & 0.17 \\
\hline
\end{tabular}

*Resolution estimate based on noise-free frequency computation interval

**Tentative identification with Gleissberg period

TABLE 1B. Carbonate Percent MAXENT* spectrum

\begin{tabular}{ccc}
\hline Period $(\mathrm{yr})$ & Frequency $\left(\mathrm{yr}^{-1}\right)$ & Rel. PSD $\left(\%^{2}-\mathrm{yr}\right)$ \\
\hline $3500^{*}$ & 0.00029 & $1.04 \times 10^{5}$ \\
$1110^{*}$ & 0.0009 & $10^{4}$ \\
$624^{*}$ & 0.0016 & 5050 \\
$456^{*}$ & 0.00219 & 6825 \\
$326^{*}$ & 0.00307 & 3213 \\
$247^{*}$ & 0.00404 & 595 \\
$201^{*}$ & 0.00498 & 506 \\
$191^{*}$ & 0.00524 & 341 \\
$142^{*}$ & 0.00705 & 733 \\
$90^{* *}$ & 0.01114 & 1387 \\
$80^{* *}$ & 0.01249 & 218 \\
$57^{* *}$ & 0.01766 & 637 \\
\hline
\end{tabular}

*MEM order 150; 1 stack of 551 data

**MEM order 100; 2 stack of 225 data

**+Tentative identification with Gleissberg period

mode is directly inferred since ${ }^{14} \mathrm{C}$ is influenced by total atmospheric $\mathrm{CO}_{2}$. Deposition is thought to be correlated with temperature and, in turn, related to the $\mathrm{CO}_{2}$ content of the sea, the surface ocean and atmosphere being presumably in a steady state. Higher ocean temperature leads to exchange towards the atmosphere. The periodogram of the total carbonate discloses a period of 3.5 $\mathrm{ka}$ (Table 1B), but the exactness of this value is not especially meaningful, in view of the less than two full period cycles. On the other hand, substantial evidence for a quasiperiodic long "period" variation at $2.3 \mathrm{ka}$ in ${ }^{14} \mathrm{C}$ exists, but an exact period, and how well it fits a simple sinusoid, is uncertain (Sonett 1984; see also Tables 2A, B). Thus, to comment on whether the long period regime of the carbonate signal has a relation to that of the ${ }^{14} \mathrm{C}$ record should be postponed until a substantially longer $\mathrm{CaCO}_{3}$ record becomes available.

As noted earlier, the carbonate record should be primarily climatic, e.g., temperature-precipitationrelated and/or biogenic. Purely oceanic forcing is difficult to explain; invoking atmospheric exchange of $\mathrm{CO}_{2}$ involves the complex chemistry of the upper ocean, with respect to $\left(\mathrm{HCO}_{3}\right)^{-}$and $\left(\mathrm{CO}_{3}\right)^{2-}$ (Broecker \& Peng 1970). Consideration of the temperature-solubility problem is beyond the scope of this paper, and, in any event, may be of greater complexity than for the open global ocean. The availability of a core $\delta^{18} \mathrm{O}$ record in this sedimentary sequence might shed light on the issue of whether the carbonate concentration is temperature-forced. 
TABLE 2A. $\Delta^{14}$ C Periodogram*

\begin{tabular}{ccc}
\hline Period $(\mathrm{yr})^{*}$ & Frequency $\left(\mathrm{yr}^{-1}\right)$ & PDS $\left(\% o^{2}-\mathrm{yr}\right)$ \\
\hline 2260 & 0.0010 & 15.60 \\
$1010^{* *}$ & 0.0010 & 2.33 \\
$700^{* *}$ & 0.0014 & 2.43 \\
$530^{* *}$ & 0.0019 & 2.28 \\
390 & 0.0025 & 1.41 \\
260 & 0.0039 & 1.27 \\
205 & 0.0049 & 2.05 \\
156 & 0.0064 & 0.47 \\
125 & 0.0080 & 0.96 \\
$88^{\dagger}$ & 0.0114 & 0.45 \\
$84^{\dagger}$ & 0.0119 & 0.34 \\
\hline
\end{tabular}

*Smoothing by 3 data points boxcar

** Suspected harmonic of $2260 \mathrm{yr}$

'Tentative identification with Gleissberg period

TABLE 2B. $\Delta{ }^{14}$ C MAXENT Spectrum

\begin{tabular}{ccc}
\hline Period $(\mathrm{yr})$ & Frequency $\left(\mathrm{yr}^{-1}\right)$ & PSD $\left(\% 0^{2}-\mathrm{yr}\right)$ \\
\hline $2400^{* * *}$ & 0.00042 & $1.04 \times 10^{5}$ \\
$2023^{* * *}$ & 0.00049 & $1.88 \times 10^{5}$ \\
$86^{* \dagger}$ & 0.00116 & $3.98 \times 10^{4}$ \\
$537^{* \dagger}$ & 0.00186 & 8640 \\
$439^{* \dagger}$ & 0.00228 & 8163 \\
$359^{* \dagger}$ & 0.00279 & 5222 \\
$278^{* \dagger}$ & 0.00358 & 3617 \\
$244^{* \ddagger}$ & 0.00409 & 3246 \\
$204^{*}$ & 0.00488 & $1.71 \times 10^{4}$ \\
$183^{* \ddagger}$ & 0.00546 & 4817 \\
$156^{*}$ & 0.00640 & 1667 \\
$126^{*}$ & 0.00791 & 5780 \\
$86^{8}$ & 0.01166 & 6665 \\
$52^{1}$ & 0.01938 & 2933 \\
\hline
\end{tabular}

*MEM order 150; stack of 2-450 data each

**Possible MEM-forced line splitting

'Possible harmonic of 2 ka line(s)

' 5 Side band of 204-yr line(?)

Tentative identification with Gleissberg period

'MEM order 100; stack of 3-303 data each

\section{The 200-Year Spectral Feature (Suess 'Wiggles')}

That a 200-yr period is present in the ${ }^{14} \mathrm{C}$ spectrum (Tables 2A, B) is now well established (Suess 1980; Sonett \& Suess 1984; Sonett 1984; Sonett \& Finney 1990). Although the source of the 200yr period is uncertain, it is thought to be solar. ${ }^{10} \mathrm{Be}$ and ${ }^{14} \mathrm{C}$ variations at periods of $10^{2}-10^{3} \mathrm{yr}$ are very well correlated (Beer et al. 1988) and therefore, thought to be primarily driven by solar variations. The 200-yr line is not detected in the spectrum of Arctic ${ }^{10} \mathrm{Be}$, but may be blurred by ice compaction. The ${ }^{14} \mathrm{C}$ spectrum shows the 200 -yr line joined by lines at 244 and $183 \mathrm{yr}$ in 
proximity to sideband positions expected if the 200-yr line were modulated by the very long period of $2.3 \mathrm{ka}$. Indeed, the narrow-banded ${ }^{14} \mathrm{C}$ record suggests such modulation (Sonett 1984; Sonett \& Finney 1990). The carbonate sequence reveals weaker evidence for this ensemble of lines (Table 1B), although the earlier, shorter Ionian Sea sequences, GT14 and GT89/3, show stronger evidence for the 200-yr period (Cini Castagnoli et al. 1990a, b). Whereas the evidence from the Campito Mt. tree-ring data supports the view that the 200 -yr period is jointly solar radiative and electrodynamic (Sonett \& Suess 1984), the carbonate evidence is mixed, though this component may be a non-persistent period.

\section{The Gleissberg Period}

Whereas spectral activity in both the ${ }^{14} \mathrm{C}$ and carbonate records at periods shorter than $100 \mathrm{yr}$ is weak, both records show the Gleissberg period. MEM is extremely sensitive to small power, but notorious for line splitting, noise and frequency shifting, as well as difficulty in determining optimum computational order. Figures $5 \mathrm{~A}$ and $\mathrm{B}$ show the high-frequency spectra for both carbonate (order 80 ) and ${ }^{14} \mathrm{C}$ (order 150). In both cases, the numerous low-amplitude lines should be regarded with care. Note the three high-frequency lines in the ${ }^{14} \mathrm{C}$ spectrum at $0.008 \mathrm{yr}^{-1}(126 \mathrm{yr}$ ), the Gleissberg at $0.0115 \mathrm{yr}^{-1}(87 \mathrm{yr})$ and $0.0195 \mathrm{yr}^{-1}(52 \mathrm{yr})$. Corresponding lines are present in the carbonate spectrum at $0.007 \mathrm{yr}^{-1}(142 \mathrm{yr}), 0.0111 \mathrm{yr}^{-1}(90 \mathrm{yr})$ and $0.0177 \mathrm{yr}^{-1}(57 \mathrm{yr})$. We expect that the first and last noted in the carbonate spectrum cannot be matched to those of the ${ }^{14} \mathrm{C}$ spectrum, but that fundamental resolution limits, together with computational errors, provide the leeway necessary to match the 87 - and 90 -yr lines.

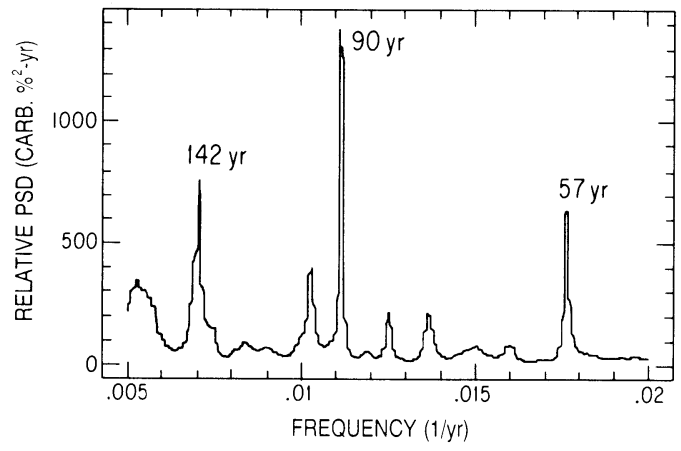

Fig. 5A. MEM (order 80) high-frequency spectrum of GT90/3 showing periods at 142,90 and $57 \mathrm{yr}$.

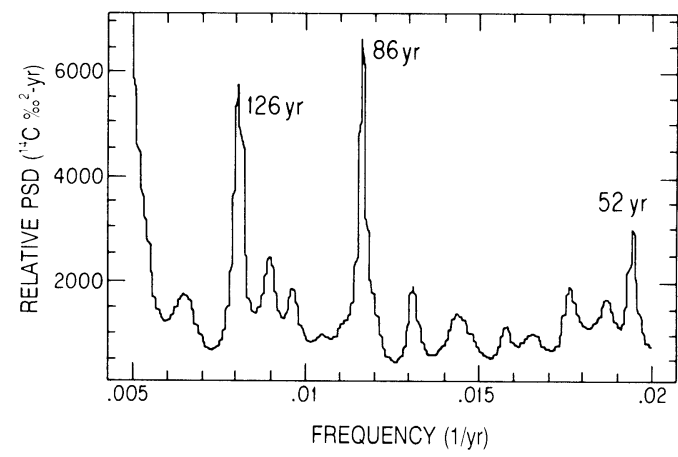

Fig. 5B. MEM (order 150) stacked high-frequency spectrum of ${ }^{14} \mathrm{C}$. Major lines are at 126,86 and $52 \mathrm{yr}$.

The study (by the method of superposition of epochs) of the phases of the sinusoid at $\mathrm{T}=88 \mathrm{yr}$ of both carbonate and ${ }^{14} \mathrm{C}$ time series gives the result that ${ }^{14} \mathrm{C}$ lags by $\mathrm{T} / 12$, about seven years, the carbonate confirming a possible climatic effect on both these records. Thus, the carbonate content time sequence appears to record the Gleissberg period (Gleissberg 1944, 1966). Presuming the carbonate record to be wholly a climatic response, we infer that the Gleissberg period is a combined electrodynamic and radiative variation. Sonett and Suess (1984) reported a similar result between tree-ring growth and ${ }^{14} \mathrm{C}$. A periodicity of $80-100$ yr was found also in rainfall in England, in the severity index of European winters, in the annual layer thickness of the Greenland ice cap, etc. (Lamb 1981: Table 6.2). The appearance of the Gleissberg period in the sunspot index identifies the period with the solar magnetic field. Detection in the aurorae shows that the periodicity is propagated in the solar wind to the Earth, affecting the magnetosphere. That this 
period also appears in the carbonate record and high-altitude tree growth suggests an associated irradiance variability.

\section{REFERENCES}

Beer, J., Siegenthaler, U., Bonani, G., Finkel, R. C., Oeschger, H. Suter, M. and Wölfli, W. 1988 Information on past solar activity and geomagnetism from ${ }^{10} \mathrm{Be}$ in the Camp Century Ice Core. Nature 331 : 675-680.

Broecker, W. S. and Peng, T. H. 1970 Tracers in the Sea. Palisades, New York, Eldigio Press: 690 p.

Cini Castagnoli, G., Bonino, G., Caprioglio, F., Provenzale, A., Serio, M. and Bhandari, N. 1990a The $\mathrm{CaCO}_{3}$ profile in a recent Ionian Sea core and the tree ring radiocarbon record over the last two millennia. Geophysical Research Letters 17: 1545-1548.

Cini Castagnoli, G., Bonino, G., Caprioglio, F., Provenzale, A., Serio, M. and Zhu Guang-Mei, 1990b The carbonate profile of two recent Ionian Sea cores: Evidence that the sedimentation rate is constant over the last millennia. Geophysical Research Letters 17: 1937-1940.

Cini Castagnoli, G., Bonino, G. and Provenzale, A. 1991 Solar-terrestrial relationships in recent sea sediments. In Sonett, C. P., Giampapa, M. S. and Matthews, M. S., eds., The Sun in Time. Tucson, The University of Arizona Press: 562-586.

Cini Castagnoli, G., Bonino, G., Provenzale, A. and Serio, M. 1992 The $\mathrm{CaCO}_{3}$ profiles of deep and shallow Mediterranean Sea cores as indicators of solar-terrestrial relationships. Nuovo Cimento $C$, in press.

Crowley, T. J. and North, G. R. 1991 Paleoclimatology. New York, Oxford University Press: 65 p.
Feynman, J. and Fougere, P. F. 1984 Eighty-eight year periodicity in solar-terrestrial phenomena confirmed. Journal of Geophysical Research 89: 3023-3027.

Gleissberg, W. 1944 A table of secular variations of the solar cycle. Terrestrial Magnetism and Atmosphere Electricity 49: 243-244.

1966 Ascent and descent in the eighty year cycle of solar activity. Journal of the British Astronomical Association 76: 265-268.

Lamb, H. H. 1981 Climate: Present, Past and Future. London, Methuen.

Sonett, C. P. 1984 Very long solar periods and the radiocarbon record. Review of Geophysics and Space Physics 22: 239-254.

1992 The present status of understanding of the long-period spectrum of radiocarbon. In Taylor, R. E., Long, A. and Kra, R. S., eds., Radiocarbon After Four Decades: An Interdisciplinary Perspective. New York, Springer-Verlag: 50-61.

Sonett, C. P. and Finney, S. A. 1990 The spectrum of radiocarbon. Philosophical Transactions of the Royal Society of London A330: 413-426.

Sonett, C. P. and Suess, H. E. 1984 Correlation of bristlecone pine ring widths with atmospheric carbon14 variations: A climate-Sun relation. Nature 307: 141-143.

Suess, H. E. 1980 The radiocarbon record in tree rings of the last 8000 years. In Stuiver, M. and Kra, R. S., eds., Proceeding of the 10th International ${ }^{14} \mathrm{C}$ Conference. Radiocarbon 22(2): 200-209. 